\title{
Chirurgie de la main: quels bénéfices pour le patient?
}

\section{Michaël Papaloïzos}

Past President et membre du Comité de la Société suisse de chirurgie de la main

Grâce aux services de la chirurgie de la main, les patients ne retrouvent pas seulement le meilleur usage possible de leur main, de leur poignet ou de leur bras, améliorant ainsi leur qualité de vie. En regagnant leurs capacités fonctionnelles quotidiennes, professionnellement ou à domicile, ils contribuent de manière positive à l'économie, directement ou indirectement.

\section{Une spécialité née de l'importance sociale et économique de la main}

La Société suisse de chirurgie de la main est née à Lausanne en 1966. Son initiateur, le Professeur Claude Verdan, comme d'autres en Europe et aux Etats-Unis, avait compris deux choses: l'importance économique de la main et du poignet - un quart de toutes les urgences, tous cas confondus - et la nécessité d'une approche spécifique car ces lésions étaient alors souvent mal traitées et nombre de patients finissaient invalides et à charge des bien-portants [1].

Cinquante ans plus tard, la chirurgie de la main a acquis le statut d'une société de discipline indépendante. Cette reconnaissance et ce développement continu se sont ainsi forgés avant tout par la plus-value que cette spécialisation a apporté aux patients comme individus et à la société en général.

\section{Un doigt sauvé peut aussi sauver une vie (de travail)}

Le mérite particulier de Verdan a été de révolutionner la chirurgie des tendons fléchisseurs. Celle-ci n'a cessé de s'améliorer depuis et permet de redonner la mobilité autrefois définitivement perdue aux doigts lésés, ceux du menuisier comme ceux du musicien.

On ne s'imagine plus si bien maintenant ce que cela signifiait alors. Verdan reçoit un soir un jeune apprenti menuisier blessé aux tendons fléchisseurs qui, bien conscient de ce qu'il encoure, lui dit: "Mes mains, c'est tout ce que j'ai, sauvez-les moi.» Les techniques de réparation comme celles de réhabilitation des tendons fléchisseurs se sont remarquablement développées et affinées depuis. De nos jours, le même patient n'aurait plus le même souci de retrouver la fonction de sa main et son aptitude au travail.

Aujourd'hui, on peut faire beaucoup plus encore comme le montre un autre exemple: une jeune pianiste de concert subit au décours d'une banale chute une fracture de la première phalange de son petit doigt, qui se trouve déformé et inutilisable. Ce genre de fracture était très difficile à traiter il y a encore deux ou trois décennies. Il en résultait le plus souvent des raideurs et des déformations définitives. Grâce aux nouveaux implants développés depuis et aux techniques de fixation qu'ils ont permis, cette patiente a été opérée, sa fracture stabilisée, permettant de rapidement mobiliser son doigt et éviter les raideurs de règle auparavant. Trois mois plus tard, elle remonte sur scène pour jouer en concert et en solo. Les quelques milliers de francs qu'auront coûté cette intervention et la réhabilitation sont de l'argent bien investi, si l'on songe qu'une carrière entière de concertiste aurait pu être perdue.

\section{Un poignet sauvé, c'est un invalide en moins}

La fracture du poignet est la fracture la plus fréquente, et touche particulièrement les femmes âgées - une tendance qui va sans doute s'accentuer encore. A la fin des années 80 , on traitait communément ces fractures en les réduisant et les plâtrant ou en posant un fixateur externe. C'était encombrant, régulièrement inefficace et source de séquelles très sérieuses aboutissant à des poignets enraidis et douloureux. Les avis étaient partagés, les directives vagues et mal fondées - bref, on nageait en eaux troubles et les bras cassés quotidiens 
des jeunes patients comme des plus âgés faisaient les frais du mauvais état des connaissances à l'époque. Les nouvelles plaques de fixation apparues au début

\section{Les nouveaux traitements permettent de redonner son indépendance au patient, qui peut ainsi être maintenu à domicile ou y retourner rapidement.}

des années 2000 ont mis tout le monde d'accord en s'imposant rapidement comme un standard incontournable dans le traitement de la plupart des fractures du poignet. Elles permettent de redonner dans de brefs délais mobilité et fonction au poignet - et indépendance au patient, qui peut ainsi être maintenu à domicile ou y retourner rapidement. Le coût d'une telle plaque (env. 1200 CHF) et de l'intervention (env. 1500 CHF selon le TARMED) est sans commune mesure avec les coûts directs et indirects d'un handicap permanent, pour les jeunes patients comme les moins jeunes.

Bien d'autres progrès ont été réalisés: microchirurgie permettant les réimplantations, endoprothèses du poignet et des articulations digitales, chirurgie mini-invasive et endoscopique, etc. - la liste serait longue s'il fallait tout énumérer. Bien plus que les nouveaux outils, c'est l'usage opportun qu'on en fait qui importe - et pour lequel une formation spécialisée est essentielle.

\section{Une spécialité en plus afin de garantir aux patients une qualité optimale}

Recourir directement à un spécialiste en chirurgie de la main, que ce soit dans l'urgence ou électivement, c'est la garantie pour le patient d'un traitement adapté, non seulement à la pathologie, mais aussi à la personne en lui proposant le traitement individualisé qui répond le mieux à ses besoins. On ne traitera pas pareillement une arthrose de la base du pouce consécutive à une fracture chez un homme de 35 ans et la même arthrose de nature dégénérative d'une femme de 65 ans. Les deux constituent un handicap certain mais dans des contextes différents qu'il faut évaluer et dont le choix thérapeutique doit tenir compte.

Il a été bien montré qu'une prise en charge inappropriée par des non-spécialistes donnait significativement de moins bons résultats et coûtait finalement plus cher [2]. De même, le recours précoce au spécialiste permet souvent la meilleure orientation thérapeutique [3].
Un bon exemple en est celui de la tendinite de Quervain, une tendinite fréquente du poignet. Il n'est pas rare qu'un chirurgien de la main reçoive un patient suivi depuis des mois pour une telle tendinite, traitée par des dizaines de séances de physiothérapie, des attelles (plus ou moins adaptées), et quelquefois après de coûteux examens, en arrêt de travail et en quasi-dépression depuis des semaines "parce qu'on ne sait pas ce que j'ai». Tout cela alors qu'un examen clinique suffit au diagnostic dans la plupart des cas et qu'une injection locale d'anti-inflammatoires ou une intervention chirurgicale ambulatoire simple mais précise assure la

La chirurgie de la main rétablit les aptitudes fonctionnelles et l'autonomie des patients et réduit en conséquence les coûts d'un handicap permanent.

guérison en trois à quatre semaines - à moindre frais. En général, pour la chirurgie de la main, le transfert de l'hospitalier à l'ambulatoire est déjà une réalité: 80 à $85 \%$ des interventions des praticiens installés sont ambulatoires. Ce taux est plus faible pour les hôpitaux qui assument une proportion plus importante de pathologies et de patients plus lourds. Cependant, avec un financement uniforme des prestations ambulatoires et hospitalières et une bonne tarification, ce taux pourrait sans doute être encore plus élevé - au bénéfice de tous.

\section{Résumé}

Que ce soit dans les suites d'un accident, qu'il s'agisse d'une entorse apparemment banale ou d'une plaie manifestement sérieuse, qu'ils souffrent d'arthrose ou de douleurs chroniques, qu'ils soient jeunes ou vieux, les patients trouveront auprès du chirurgien de la main l'assurance de la meilleure prise en charge possible - en premier comme en ultime recours. Les diagnostics et les traitements par des spécialistes sont à même de soulager les patients de manière optimale et d'améliorer leur qualité de vie. Au-delà, la chirurgie de la main rétablit l'aptitude au travail et l'autonomie des patients et en conséquence les coûts directs et indirects d'un handicap permanent.

\section{Références}

1 Dias JJ, Garcia-Elias M. Hand injury costs. Injury, Int J Care Injured. 2006;37:1071-7.

2 Kenesi C, Masmejean E. Les urgences main en France. Un important défi de santé publique. Bull Acad Natl Med. 2004;188:793-800.

3 Raimbeau G. Coûts des urgences mains. Chir Main. 2003;22:258-63. 